\title{
Cryptogenic brain abscess due to community-associated methicillin-resistant Staphylococcus aureus
}

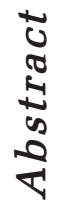

\section{Deepak Juyal, Shekhar Pal, Mahesh Ramola 1 , Vyas Kumar Rathaur', Neelam Sharma, Anurag Ayachit ${ }^{3}$}

Departments of Microbiology and Immunology, ${ }^{1}$ Neurosurgery, ${ }^{2}$ Pediatrics, Veer Chandra Singh

Garhwali Government Medical Sciences and Research Institute, Srinagar Garhwal, Uttarakhand,

${ }^{3}$ Department of Radiodiagnosis and Imaging, Kasturba Medical College, Manipal, Karnataka, India

Address for the Correspondence: Mr. Deepak Juyal, Department of Microbiology and Immunology, Veer Chandra Singh Garhwali Govt Medical Sciences and Research Institute, Srinagar Garhwal - 246 174, Uttarakhand, India.

E-mail: deepakk787@gmail.com

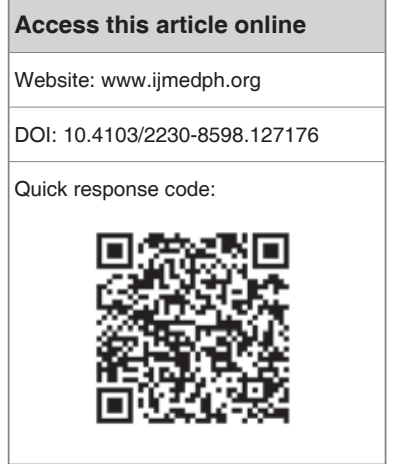

We report a case of cryptogenic brain abscess caused by infection due to communityassociated methicillin-resistant Staphylococcus aureus (CA-MRSA) in a previously healthy, 2-year-old, girl child with a history of persistent headache and fever for several weeks. Headache acutely worsened the day before hospital admission with vomiting, right hemiparesis, and altered sensorium. Community-onset pyogenic brain abscess should be added to the growing list of life-threatening invasive infections caused by community-acquired S. aureus. Early diagnosis, prompt neurosurgical drainage, and appropriate medical therapy are important for management of such infections. In light of expanding community-acquired $S$. aureus epidemic and the life-threatening nature of the disease we recommend empirical use of vancomycin in all cases of community onset brain abscess.

Key words: MRSA, pyogenic brain abscess, Staphylococcus aureus, vancomycin

\section{INTRODUCTION}

Staphylococcus aureus is among the most important pathogen because of both the diversity and the severity of infections it causes. Methicillin resistant $S$. aureus (MRSA) was first described in $1961^{[1]}$ and since then has become a significant pathogen in nosocomial infections. Although historically considered as typical nosocomial pathogen, MRSA has rapidly emerged as a causative agent for community infections. ${ }^{[2]}$ Risk factors for community acquisition are recent or frequent hospital admissions, frequent antibiotic exposure, chronic illness, prior surgery or a carrier in the family. ${ }^{[3]}$ Recently, patients with no risk factors are presenting with community-associated MRSA (CA-MRSA) infections. ${ }^{[4]}$ For clinicians, the spread of such methicillin resistant strains is a matter of concern as the therapeutic outcome of infections caused by MRSA is worse than that of caused by methicillin sensitive $S$. aureus. ${ }^{[5]}$ In India the prevalence of infections due to CA-MRSA appears to be lower than the western countries, although recent reports highlight that these infections are on rise.

CA-MRSA harbors a Staphylococcal cassette chromosome mec (SCCmec) element that is distinct from those found in healthcare associated MRSA (HA-MRSA) strains and typically carries the PantonValentine leukocidin (PVL) toxin genes ${ }^{[6]}$ encoding a toxin endowed with the ability to kill leukocytes.

Here we present a case of cryptogenic brain abscess caused by CA-MRSA in a previously healthy child.

\section{CASE REPORT}

A 2-year-old girl child was brought to the emergency department of our hospital with a history of persistent headache and fever for several weeks. Headache acutely worsened the day before hospital admission associated with vomiting, right sided weakness, and altered sensorium (as noted by the parents). Initial examination showed temperature $39^{\circ} \mathrm{C}$, heart rate 95 beats $/ \mathrm{min}$, respiratory rate $35 \mathrm{breaths} / \mathrm{min}$, and blood pressure 120/85 mm Hg. The patient was too irritable to the central nervous system (CNS) examination to be done. Neurological examination confirmed right sided hemiparesis with anisocoria and mild disorientation (Glasgow coma scale 12/15). General physical examination was normal and pertinent negative findings included no adventitial lung sounds; no heart murmurs; abdomen was soft without 
any organomegaly; extremities were normal; and no cutaneous rash, sore, or abscess was noted. A full sepsis profile and a brain computed tomography (CT) scan was requested.

Brain CT scan revealed multiple small to large, coalescing, hypodense parenchymal lesions [Figure 1a]; largest measuring about $5 \times 5 \mathrm{~cm}$ in size [Figure 1b] in the left temporal, frontal, and parietal regions. The lesions had peripherally enhancing thin-walled collections with extensive perilesional vasogenic edema, resultant mass effect, and diffuse cerebral edema. Significant midline shift to the right was noted with subfalcine herniation [Figure 1c], compression of left lateral and third ventricle. Dilatation of right lateral ventricle was seen and significant mid brain rotation was also noted. The findings were suggestive of multiple ring enhancing lesions and cerebral abscess.

Blood work up showed a white blood cell (WBC) count of 19,500 cells $/ \mathrm{ml}$ with $89 \%$ polymorphonuclear leukocytes (PMN's) and hemoglobin $12.6 \mathrm{gm} / \mathrm{dl}$. Serum C-reactive protein $(24 \mathrm{mg} / \mathrm{dl})$ and procalcitonin (8 U/l) levels were elevated. Arterial blood gases and chest X-ray were normal. Lumbar puncture was not done to avoid any possible herniation. Empirical treatment with intravenous (IV) ceftazidime and metronidazole was started and patient was transferred to the neurosurgical intensive care unit (ICU). The patient was taken emergently to the operating room for surgical decompression and abscess evacuation through a left temporal burr hole under general anesthesia and about $120 \mathrm{ml}$ of thick yellow pus was evacuated which was immediately sent for microbiological investigations. Gram stain of the aspirated pus showed plenty of PMN's and many gram positive cocci in clusters. IV vancomycin was added to the regime.

Two days following admission, the aspirated specimen yielded a pure growth of MRSA, susceptible to tetracycline, clindamycin, gentamicin, cotrimoxazole, and vancomycin; and resistant to methicillin, erythromycin, ciprofloxacin, penicillin, and ampicillinsulbactam. Same treatment was continued. Anaerobic culture of the aspirated pus and blood samples collected before the administration of antibiotics yielded no bacterial growth.

The postoperative course was uneventful without any clinical or laboratory evidence of infection. Aspiration of the abscess was repeated on $9^{\text {th }}$ and $17^{\text {th }}$ day of the therapy and culture of these specimens were negative. Nasal swabs taken for infection control measure were negative for $S$. aureus. Parenteral antibiotic therapy was continued for 3 weeks and was followed by 8 weeks course of teicoplanin (800 $\mathrm{mg}$ thrice/week). During this treatment, the patient remained afebrile and there were no signs of infection at a 6 month follow-up examination. CT scan taken at this point revealed no obvious abscess [Figure 2].

\section{Characterization of MRSA}

S. aureus was identified by standard microbiological methods. Antibiotic susceptibility testing was performed by Kirby-Bauer disk diffusion method on Muller-Hinton agar (HiMedia Laboratories Pvt. Ltd, India) supplemented with $2 \% \mathrm{NaCl}$. Methicillin resistance was confirmed using PBP2a latex agglutination test (Oxoid Ltd, Hampshire, UK) and then by polymerase chain reaction (PCR) assay targeting mecA gene. Further characterization of the organism was obtained by PCR assay for SCCmectype and presence of PVL genes lukS-PV and lukF-PV. The isolate carried SCCmec type IV and genes coding PVL confirming it to be a CA-MRSA strain. The results of the D-test were negative for inducible clindamycin resistance.

\section{DISCUSSION}

S. aureus is estimated to cause $10-21 \%$ of all pyogenic brain abscesses ${ }^{[7]}$ and the pathogenesis of brain abscess is largely comprehended in terms of the route of pathogen dissemination to brain. Infection most commonly occurs after direct inoculation during neurosurgical procedures, cranial trauma, from contagious foci such as sinusitis, mastoiditis, and facial or scalp infections. ${ }^{[7]}$ Hematogenous dissemination of $S$. aureus from a peripheral source (pneumonia or endocarditis) is
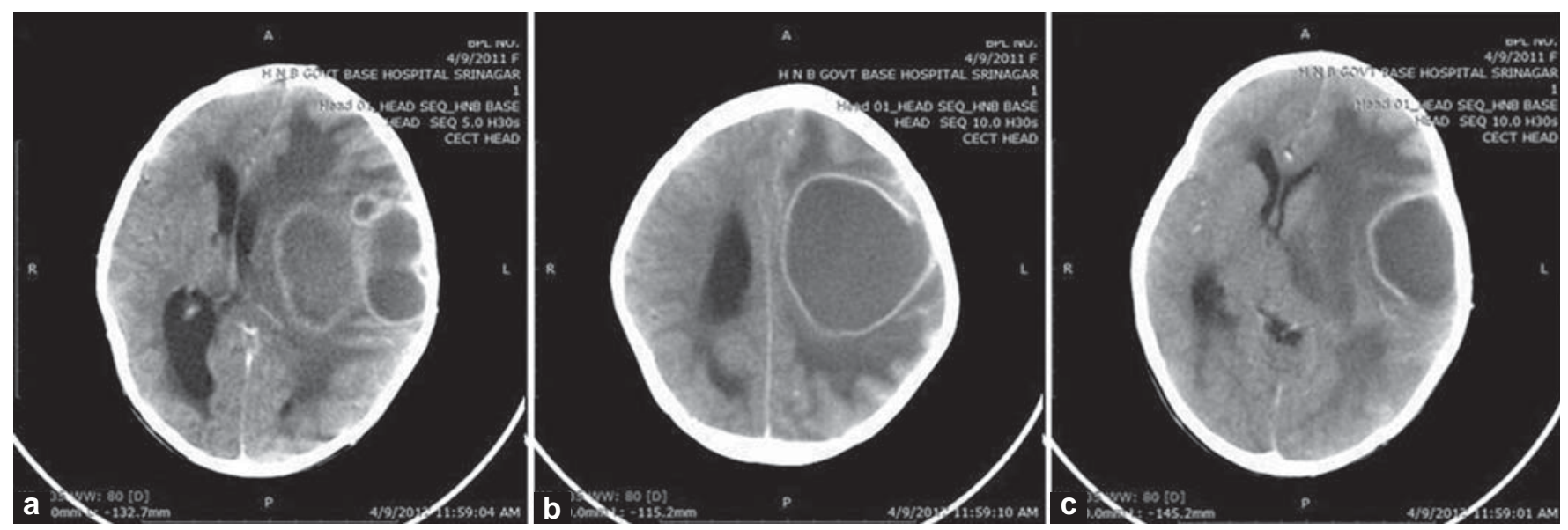

Figure 1: Preop computed tomography (CT) scan pictures. (a) Shows multiple, lobulated, hypodense, coalescing lesions in the left cerebral hemisphere with extensive perilesional vasogenic edema. (b) At level of corona radiata. Shows lobulated, hypodense, parenchymal lesion in the left frontal-parietal region with marked vasogenic edema surrounding it. (c) At level of foramen of Monro. Shows a similar well defined left temporal lesion with isodense thin wall, significant midline shift to the right with subfalcine herniation and midbrain rotation 


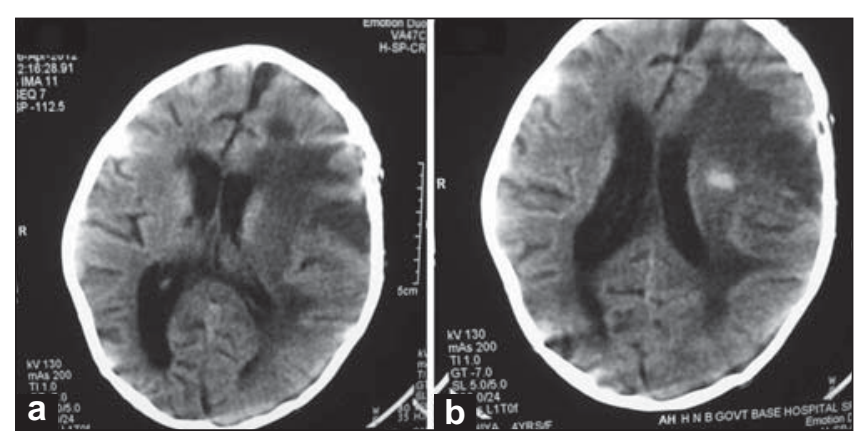

Figure 2: Postop CT scan pictures. (a, b) Follow-up CT scan shows no obvious abscess, mild residual midline shift, prominence of ventricular system, and residual calcification.

an infrequent manifestation because of the high intrinsic resistance of the blood brain barrier to infection. ${ }^{[7]}$ In a subset of cases there is an underlying medical condition such as congenital heart disease, human immunodeficiency virus (HIV), IV drug use, diabetes, or alcoholism. ${ }^{[8]}$ In $20-30 \%$ of cases no source of infection can be identified and these are considered as cryptogenic. ${ }^{[7]}$ The development of a serious infections in children without predisposing conditions is a typical feature of CA-MRSA. The most common infections resulting from CA-MRSA involve skin and soft tissue, though cases of fatal, invasive infections have occurred in children ${ }^{[9]}$ and only recently, CA-MRSA was found to cause CNS infections. Further, CA-MRSA are often PVL positive ${ }^{[6]}$ and infections with such strains are associated with higher mortality. ${ }^{[0]}$

Diagnosis in our case was based on clinical investigation, neuroimaging, and positive abscess aspirate culture. Laboratory results were supported by neurological findings and brain CT. On neurological examination the classic triad of fever, headache, and focal neurological deficits were present. Signs and/or symptoms of raised intracranial pressure including nausea, vomiting, and papilledema were present.

Outcomes for patients with CA-MRSA brain abscesses have not been thoroughly studied. The overall mortality rate for brain abscesses is decreasing; however, rupture of the brain abscess into the ventricular system remains a devastating complication often resulting in death. ${ }^{[8]}$ The prompt diagnosis, timely surgical abscess evacuation, and appropriate therapy lead to the successful treatment of our patient. The patient showed progressive improvement and at follow-up 6 months later was doing well. However, authors were not able to determine how the patient acquired MRSA. In previous studies, the patent foramen ovale (PFO) has been suggested to be a potential source of embolism leading to cryptogenic stroke. There have been four reported cases describing an association between cryptogenic brain abscess and PFO ${ }^{[10]}$ Proposed explanations for this association include poor dental hygiene resulting in systemic oral flora to gain access into the arterial system via a PFO. Alternatively, PFO may be a predisposing factor to hematogenous spread from a primary silent focus.

\section{CONCLUSION}

S. aureus is a common cause of pyogenic brain abscess. The pathogenesis and clinical features of the Staphylococcal brain abscess do not differ from pyogenic brain abscess caused by other microorganisms. This observation highlights the importance of obtaining abscess aspirate and/or biopsy specimens for specific microbiological diagnosis. Optimal management of pyogenic brain abscess includes a combination of prompt neuroimaging, neurosurgical drainage, and appropriate medical therapy. A combination of third generation cephalosporin and metronidazole has traditionally been recommended for empirical treatment of community-onset brain abscess. In light of the expanding CA-MRSA epidemic and life-threatening nature of the disease we recommend empirical use of vancomycin in all cases of community onset brain abscess potentially caused by $S$. aureus infection pending culture and in vitro susceptibility test results, even in patients without clear risk factors for CA-MRSA infection. Similar recommendations have been made by other authors. ${ }^{[7,11]}$

\section{Consent}

A written informed consent was obtained from the patient's parents for publication of this case report and of the accompanying image.

\section{REFERENCES}

1. Jevons MP. ‘Celbenin'-resistant staphylococci. Br Med J 1961;1:124-5.

2. Naesens R, Ronsyn M, Druwé P, Denis O, leven M, Jeurissen A. Central nervous system invasion by community-acquired meticillin-resistant Staphylococcus aureus. J Med Microbiol 2009;58:1247-51.

3. Salgado CD, Farr BM, Calfee DP. Community-acquired methicillin resistant Staphyloccus aureus: A meta-analysis of prevalence and risk factors. Clin Infect Dis 2003;36:131-9.

4. Sattler CA, Mason EO Jr, Kaplan SL. Prospective comparison of risk factors and demographic and clinical characteristics of communityacquired, methicillin-resistant versus methicillin-susceptible Staphylococcus aureus infection in children. Pediatr Infect Dis J 2002;21:910-7.

5. Cosgrove SE, Sakoulas G, Perencevich EN, Schwaber MJ, Karchmer AW, Carmeli Y. Comparison of mortality associated with methicillinresistant and methicillin-susceptible Staphylococcus aureus bacteremia: A meta-analysis. Clin Infect Dis 2003;36:53-9.

6. Boyle-Vavra S, Daum RS. Community-acquired methicillin-resistant Staphylococcus aureus: The role of Panton-Valentine leukocidin. Lab Invest 2007;87:3-9.

7. Tunkel AR. Brain abscess. In: Mandell GL, Bennett JE, Dolin R, editors Mandell, Douglas, and Bennett's Principles and Practice of Infectious Diseases. $6^{\text {th }}$ ed. Philadelphia: Elsevier/Churchill Livingstone; 2005. p. 1150-63.

8. Carpenter J, Stapleton S, Holliman R. Retrospective analysis of 49 cases of brain abscess and review of the literature. Eur $\mathrm{J}$ Clin Microbiol Infect Dis 2007;26:1-11.

9. Nourse C, Starr M, Munckhof W. Community-acquired methicillin-resistant Staphylococcus aureus causes severe disseminated infection and deep venous thrombosis in children: Literature review and recommendations for management. J Paediatric Child Health 2007; 43:656-61.

10. Khouzam RN, El-Dokla AM, Menkes DL. Undiagnosed PFO presenting as a cryptogenic brain abscess: Case report and review of literature. Heart Lung 2006;35:108-11.

11. Sifri CD, Park J, Helm GA, Stemper ME, Shukla SK. Fatal brain abscess due to community-associated methicillin-resistant Staphylococcus aureus strain USA300. Clin Infect Dis 2007;45:e113-7.

How to cite this article: Juyal D, Pal S, Ramola M, Rathaur VK, Sharma N, Ayachit A. Cryptogenic brain abscess due to communityassociated methicillin-resistant Staphylococcus aureus. Int J Med Public Health 2014;4:134-6.

Source of Support: Nil, Conflict of Interest: None declared. 